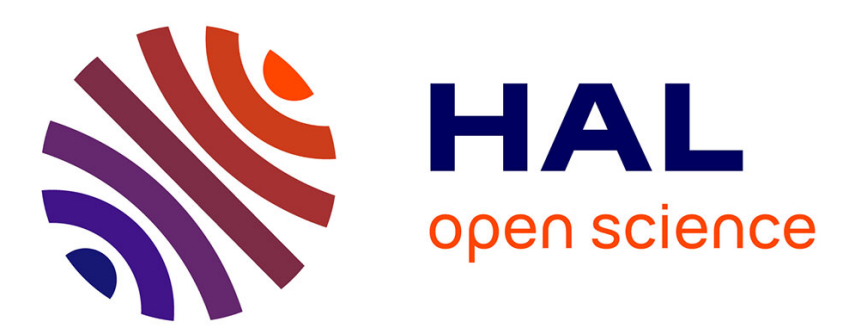

\title{
A note on 2-input neoclassical production functions
}

Gwenaël Moysan, Mehdi Senouci

\section{To cite this version:}

Gwenaël Moysan, Mehdi Senouci. A note on 2-input neoclassical production functions. Journal of Mathematical Economics, 2016, 67, pp.80-86. 10.1016/j.jmateco.2016.09.011 . hal-01383290

\section{HAL Id: hal-01383290 \\ https://hal.science/hal-01383290}

Submitted on 18 Oct 2016

HAL is a multi-disciplinary open access archive for the deposit and dissemination of scientific research documents, whether they are published or not. The documents may come from teaching and research institutions in France or abroad, or from public or private research centers.
L'archive ouverte pluridisciplinaire $\mathbf{H A L}$, est destinée au dépôt et à la diffusion de documents scientifiques de niveau recherche, publiés ou non, émanant des établissements d'enseignement et de recherche français ou étrangers, des laboratoires publics ou privés.

\section{(이) $\$$}

Distributed under a Creative Commons Attribution - NonCommercial - NoDerivatives 44.0 


\title{
A note on 2-input neoclassical production functions*
}

\author{
Gwenaël Moysan $^{\dagger} \quad$ Mehdi Senouci ${ }^{\ddagger}$
}

September 26, 2016

\begin{abstract}
In this short note, we show how the space of elasticity of substitution functions maps into the space of 2-input neoclassical production functions. In doing so we derive a general analytical formula for every 2-input neoclassical production function of class $\mathscr{C}^{2}$. We present a simple set of sufficient conditions for the Inada conditions to hold; and prove that the Solow model under capital-augmenting (or investment-specific) technical change is asymptotically balanced if and only if the capital share converges to a non-degenerated limit as the capital-labor ratio tends to infinity.
\end{abstract}

Keywords: Production function, elasticity of substitution, capital share, labor share, Solow model.

\section{Introduction}

Production functions constitute the cornerstone of supply side economics; still, perhaps surprisingly, the economist's standard toolbox contains very few of these. The economist's toolbox contains the often used Cobb-Douglass specification as well as the more recently popular CES functions. Some variable elasticity of substitution (VES) production functions have come up - see for example Revankar (1971) - but their use has remained marginal in theoretical as well as in empirical literature. Recent evidence on the existence of medium-run growth regimes makes the design of new production functions an issue of particular interest to growth theory and empirics. ${ }^{1}$

\footnotetext{
*This paper was written while Gwënael Moysan was $\mathrm{PhD}$ student at Ecole Normale Supérieure de Lyon and while Mehdi Senouci was PhD student at Paris School of Economics and Attaché Temporaire d'Enseignement et de Recherche at Ecole Normale Supérieure de Lyon. The results in this paper were part of Mehdi Senouci's PhD thesis. An earlier version of this paper circulated under the title "A general characterization of neoclassical production functions and an application to physical capital-based growth models". The authors are grateful to an anonymous referee, Antoine d'Autume, Daniel Cohen, Rodolphe dos Santos Ferreira, Patrick Pintus, Gilles Saint-Paul, Robert M. Solow and Bertrand Wigniolle, as well as to conference participants at the Augustin Cournot Doctoral Days 2014 and seminar participants at the Paris School of Economics and at Universitat de Barcelona (Department of Economic Theory) for useful comments and suggestions on earlier drafts. All remaining errors are our own.

${ }^{\dagger}$ Global Market Solutions (contact: Global Market Solutions R\&D center, 7 Cité de l'Ameublement, 75011 Paris (FRANCE), gwenael.moysan@globms.com).

${ }^{\ddagger}$ Université Paris-Saclay/CentraleSupélec, Laboratoire Genie Industriel (contact: CentraleSupélec (Office C428), Grande Voie des Vignes, 92290 Chatenay-Malabry (FRANCE). mehdi.senouci@centralesupelec.fr).

${ }^{1}$ See Greenwood and Yorukoglu (1997), Greenwood et al. (1997) and Neiman and Karabarbounis (2014).
} 
The prime goal of this paper is to derive an analytical formula for every 2-input neoclassical production function $(f)$ of class $\mathscr{C}^{2}$. The method that we present rests on the elasticity of substitution functions $(\sigma)$. We prove that for any continuous function of $\mathbb{R}_{+}^{*}$ into itself $\sigma$ there exists a 2-input neoclassical production function $(f)$ of class $\mathscr{C}^{2}$ - which is unique up to the choice of two constants - such that $\sigma$ is the elasticity of substitution function associated to $f$. In short, we prove that the space of elasticity of substitution functions is exactly the space of continuous functions of $\mathbb{R}_{+}^{*}$ into itself, and we map the space of elasticity of substitution functions into the space of neoclassical production functions through an integral formula.

The rest of the paper is organized as follows. In section 1 we derive the general formula for 2 -input production functions of class $\mathscr{C}^{2}$. In section 2 we focus on two types of production functions that are important for pure neoclassical growth theory: the production functions that satisfy the Inada conditions, and the production functions for which the Solow model under capitalaugmenting (or investment-specific) technical change is asymptotically balanced. Section 3 concludes.

\section{A general formula for neoclassical production functions}

A neoclassical production function with $n \geq 2$ inputs is a constant-returns to scale function of class $\mathscr{C}^{2} F:\left(\mathbb{R}_{+}^{*}\right)^{n} \rightarrow \mathbb{R}_{+}^{*},\left(X_{1}, X_{2}, \ldots, X_{n}\right) \mapsto F\left(X_{1}, X_{2}, \ldots, X_{n}\right)$ satisfying the assumption of strictly positive and strictly decreasing marginal returns to each input: $\forall i \in\{1, \ldots, n\}, \frac{\partial F}{\partial X_{i}}>0, \frac{\partial^{2} F}{\partial X_{i}^{2}}<0$.

In the case of two inputs $(n=2)$, and by labelling $X_{1}=K$ and $X_{2}=L$, the property of constant returns to scale yields the intensive form of $F: \forall K, L>0, F(K, L)=L f\left(\frac{K}{L}\right)$, with $f(\cdot)=F(\cdot, 1)$. These assumptions of strictly positive and strictly diminishing marginal returns on $F$ are equivalent to the following assumptions on the intensive form $f$ of $F: f^{\prime}>0, f^{\prime \prime}<0$. Further these assumptions on $F$ are sufficient to guarantee the concavity of $F$. This preliminary result is proven in appendix A.1; for the rest of the paper we focus on intensive forms.

We denote by $\mathscr{N}$ the set of 2-input neoclassical production functions in intensive form: $\mathscr{N}=$ $\left\{f: \mathbb{R}_{+}^{*} \longrightarrow \mathbb{R}_{+}^{*}, \mathscr{C}^{2} \mid f^{\prime}>0, f^{\prime \prime}<0\right\}$.

For any function $f \in \mathscr{N}$, at any capital-labor ratio $k$, we can define the elasticity of substitution of $f$ at $k$ by:

$$
\sigma(k)=-\frac{f^{\prime}(k)\left(f(k)-k f^{\prime}(k)\right)}{k f(k) f^{\prime \prime}(k)}
$$

Let $\mathfrak{S}$ be the following space of functions: $\mathfrak{S}=\left\{\sigma: \mathbb{R}_{+}^{*} \longrightarrow \mathbb{R}_{+}^{*}, \mathscr{C}^{0}\right\}$. We prove below that $\mathfrak{S}$ is precisely the space of admissible elasticity of substitution functions.

The relationship (1) can be inverted. Let $f \in \mathscr{N}$ and $\alpha(\cdot)$ be the corresponding capital share function and $\Pi(\cdot)$ the relative factor shares function: $\forall k>0, \alpha(k)=\frac{k f^{\prime}}{f} \in(0,1)$ and $\Pi(k)=\frac{\alpha(k)}{1-\alpha(k)}=$ $\frac{k f^{\prime}}{f-k f^{\prime}}>0$. The derivative function of $\Pi(\cdot)$ is linked to the elasticity of substitution through the following equation: 


\section{Lemma 1.}

$$
\frac{\Pi^{\prime}(k)}{\Pi(k)}=\frac{\sigma(k)-1}{\sigma(k)} \frac{1}{k}
$$

Proof.

$$
\frac{d \ln \Pi(k)}{d k}=\frac{d \ln \frac{k f^{\prime}}{f-k f^{\prime}}}{d k}=\frac{1}{k}+\frac{f^{\prime \prime}}{f^{\prime}}-\frac{-k f^{\prime \prime}}{f-k f^{\prime}}=\frac{1}{k}\left(1-\frac{f-k f^{\prime}}{f} \frac{1}{\sigma}-\frac{k f^{\prime}}{f} \frac{1}{\sigma}\right)=\frac{\sigma-1}{\sigma} \frac{1}{k} .
$$

As is well known, when $\sigma(k)<1$ (resp. $\sigma(k)>1$ ), $\alpha$ and $\Pi$ decrease (resp. increase) in response to capital deepening around $k$.

Let's take $\bar{k}>0$ to be some reference capital-labor ratio - for example, $\bar{k}=1$ - and let's denote by $\bar{\Pi}=\Pi(\bar{k})$ the corresponding relative factor shares ratio. Then, by integrating equation (2) between $\bar{k}$ and $k$, it comes that for all $k>0$ : $\Pi(k)=\bar{\Pi} \exp \left(\int_{\bar{k}}^{k} \frac{\sigma\left(k^{\prime}\right)-1}{\sigma\left(k^{\prime}\right)} \frac{\mathrm{d} k^{\prime}}{k^{\prime}}\right)$. Hence, $f$ is a solution to the differential equation: $\frac{k f^{\prime}}{f-k f^{\prime}}=\bar{\Pi} \exp \left(\int_{\bar{k}}^{k} \frac{\sigma\left(k^{\prime}\right)-1}{\sigma\left(k^{\prime}\right)} \frac{\mathrm{d} k^{\prime}}{k^{\prime}}\right)$ which can be re-arranged like:

$$
\frac{f^{\prime}}{f}=\frac{1}{k} \frac{1}{1+\frac{\exp \left(-\int_{\bar{k}}^{k} \frac{\sigma\left(k^{\prime}\right)-1}{\sigma\left(k^{\prime}\right)} \frac{\mathrm{d} k^{\prime}}{k^{\prime}}\right)}{\bar{\Pi}}}
$$

Notice that the term $1 /\left(1+\exp \left(-\int_{\bar{k}}^{k} \frac{\sigma\left(k^{\prime}\right)-1}{\sigma\left(k^{\prime}\right)} \frac{\mathrm{d} k^{\prime}}{k^{\prime}}\right) / \bar{\Pi}\right)=\frac{\Pi(k)}{1+\Pi(k)}$ is equal to the capital share $\alpha(k)$. Integrating (3), we conclude that there exists a constant $A>0$ such that, for all $k>0$ :

$$
f(k)=A \exp \left(\int_{\bar{k}}^{k} \frac{\mathrm{d} k^{\prime}}{k^{\prime}\left(1+\frac{\exp \left(-\int_{\bar{k}}^{k^{\prime}} \frac{\sigma\left(k^{\prime \prime}\right)-1}{\sigma\left(k^{\prime \prime}\right)} \frac{\mathrm{d} k^{\prime \prime}}{k^{\prime \prime}}\right)}{\bar{\Pi}}\right)}\right)
$$

We can state and prove our first main result, which maps the space of elasticity of substitution functions into the space of neoclassical production functions :

Theorem 1. Let $\bar{k}>0$ be some reference capital-labor ratio, and let $\bar{\Pi}>0$ and $A>0$ be two constants. Then, for any function $\sigma \in \mathfrak{S}$, there exists one and only one production function $f \in \mathscr{N}$ such that (i) $\sigma$ is the elasticity substitution function associated to $f$, and $(i i) \Pi(\bar{k})=\bar{\Pi}$ and $A=f(\bar{k})$. The formula for this function is given by (4).

Proof. Let $\sigma \in \mathfrak{S}$ and let $f$ be the function of $\mathbb{R}_{+}^{*}$ into itself defined by equation (4). By construction, if $f \in \mathscr{N}$ then $\sigma$ is the elasticity of substitution function corresponding to function $f$; so there remains only to demonstrate that $f$ defined in (4) belongs to the set $\mathscr{N}$.

Since $\sigma$ is continuous, $f$ is $\mathscr{C}^{2} . f$ is also nonnegative from (4) and for all $k>0$ :

$$
\begin{aligned}
& f^{\prime}(k)=\frac{f(k)}{k\left(1+\frac{\exp \left(-\int_{k}^{k} \frac{\sigma-1}{\sigma} \frac{\mathrm{d} k^{\prime}}{k^{\prime}}\right)}{\bar{\Pi}}\right)} \quad>0
\end{aligned}
$$

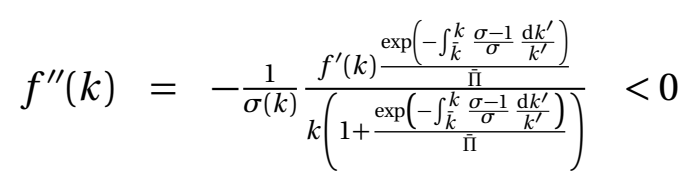


which proves that $f \in \mathscr{N}$.

Thus, all well-behaved neoclassical production functions have a representation in terms of their elasticity of substitution function, which can be any continuous function of $\mathbb{R}_{+}^{*}$ into itself. ${ }^{2}$

For example, if $\sigma \equiv 1$, equation (4) becomes: $f(k)=A \exp \left(\int_{\bar{k}}^{k} \frac{\alpha}{k^{\prime}} \mathrm{d} k^{\prime}\right)=A\left(\frac{k}{\bar{k}}\right)^{\alpha}$, where $\alpha=\frac{\bar{\Pi}}{1+\bar{\Pi}} \in$ $(0,1)$.

If $\sigma \equiv \hat{\sigma}$, with $\hat{\sigma} \neq 1$, then: $\int_{\bar{k}}^{k^{\prime}} \frac{\sigma-1}{\sigma} \frac{\mathrm{d} k^{\prime \prime}}{k^{\prime \prime}}=\frac{\hat{\sigma}-1}{\hat{\sigma}} \ln \left(\frac{k^{\prime}}{\bar{k}}\right)$. In this case, formula (4) translates into:

$$
\begin{aligned}
f(k) & =A \exp \left(\int_{\bar{k}}^{k} \frac{\mathrm{d} k^{\prime}}{k^{\prime}\left(1+\frac{\left.\left(k^{\prime}\right) \bar{k}\right)^{\frac{\sigma}{\tilde{\sigma}}}}{\bar{\Pi}}\right)}\right)=A \exp \left(\left[\frac{\hat{\sigma}}{\hat{\sigma}-1} \ln \left(\bar{k}\left(\frac{k^{\prime}}{\bar{k}}\right)^{\frac{1}{\tilde{\sigma}}}+\bar{\Pi} k^{\prime}\right)-\frac{1}{\hat{\sigma}-1} \ln k^{\prime}\right]_{k^{\prime}=\bar{k}}^{k}\right) \\
& =A\left(\frac{\bar{\Pi}}{1+\bar{\Pi}}\left(\frac{k}{\bar{k}}\right)^{\frac{\hat{\sigma}-1}{\hat{\sigma}}}+\frac{1}{1+\bar{\Pi}}\right)^{\frac{\partial}{\tilde{\sigma}-1}}
\end{aligned}
$$

Let us define $\bar{\alpha}=\frac{\bar{\Pi}}{1+\bar{\Pi}}$. The above equation yields: $f(k)=A\left(\bar{\alpha}\left(\frac{k}{\bar{k}}\right)^{\frac{\hat{\sigma}-1}{\sigma}}+1-\bar{\alpha}\right)^{\frac{\hat{\sigma}}{\sigma}}$, which is the expression of the only CES production function of elasticity parameter $\hat{\sigma}$, and such that product at $\bar{k}$ is equal to $A$ and capital share at $\bar{k}$ is $\bar{\alpha} .^{3}$

\section{Two classes of production functions}

We study how certain restrictions on function $\sigma$ ensure that the resulting production functions have some properties that are often required in economic growth models.

\subsection{The elasticity of substitution function and the Inada conditions}

The integral formula (4) permits to link the Inada conditions to the behavior of the capital share near zero and infinity.

Let $\sigma \in \mathfrak{S}$, and $\bar{k}, A$ and $\bar{\Pi}$ be strictly positive constants and let $f \in \mathscr{N}$ be the production function such that $\sigma$ is the elasticity of substitution function associated to $f$ and such that $f(\bar{k})=A$ and $\frac{\bar{k} f^{\prime}(\bar{k})}{f(\bar{k})-\bar{k} f^{\prime}(\bar{k})}=\bar{\Pi}$. Theorem 1 proves that function $f$ is unique.

$f \in \mathscr{N}$, so $f$ is strictly increasing and strictly positive over $\mathbb{R}_{+}^{*}$ while $f^{\prime}$ is strictly decreasing and strictly positive over $\mathbb{R}_{+}^{*}$. Hence, $f$ and $f^{\prime}$ admit some limits at the borders of $\mathbb{R}_{+}^{*}$. Let's denote these limits by $\left(l_{0}, l_{\infty}, l_{0}^{\prime}, l_{\infty}^{\prime}\right)$ :

$$
\begin{array}{rlrl}
l_{0} & =\lim _{0} f & \in \mathbb{R}_{+} \\
l_{\infty}=\lim _{+\infty} f & \in \mathbb{R}_{+}^{*} \cup\{+\infty\} \\
l_{0}^{\prime}=\lim _{0} f^{\prime} & \in \mathbb{R}_{+}^{*} \cup\{+\infty\} \\
l_{\infty}^{\prime}=\lim _{+\infty} f^{\prime} & \in \mathbb{R}_{+}
\end{array}
$$

The Inada conditions hold when $l_{0}=l_{\infty}^{\prime}=0$ and $l_{\infty}=l_{0}^{\prime}=+\infty$.

\footnotetext{
${ }^{2}$ Remark that equation (4) is equivalent to: $f(k)=A \exp \left(\int_{\bar{k}}^{k} \frac{\alpha\left(k^{\prime}\right)}{k^{\prime}} \mathrm{d} k^{\prime}\right)$, where $\alpha(\cdot)$ is the capital share function. This seems to be a simpler representation than (4); however, some restrictions have to be imposed on $\alpha(\cdot)$ to ensure that function $A \exp \left(\int_{\bar{k}}^{k} \frac{\alpha\left(k^{\prime}\right)}{k^{\prime}} \mathrm{d} k^{\prime}\right)$ is a neoclassical production function.

${ }^{3}$ See Arrow, Chenery, Minhas and Solow (1961), who first derived the analytical expression of two-inputs CES production function through to the same method we use here.
} 
Call $\Pi(k)=\frac{k f^{\prime}}{f-k f^{\prime}}$ the relative factor shares function and $\alpha(k)=\frac{k f^{\prime}}{f}$ the capital share function. We have seen in section 1 that for all $k>0, \Pi(k)=\bar{\Pi} \exp \left(\int_{\bar{k}}^{k} \frac{\sigma\left(k^{\prime}\right)-1}{\sigma\left(k^{\prime}\right)} \frac{\mathrm{d} k^{\prime}}{k^{\prime}}\right)$, and that $\alpha(k)=\frac{\Pi(k)}{1+\Pi(k)}$.

\section{Lemma 2.}

$$
\begin{aligned}
l_{0} & >0 \Longrightarrow \begin{aligned}
\lim _{0} \alpha & =0 \\
l_{\infty} & <+\infty
\end{aligned} \lim _{+\infty} \alpha=0 \\
l_{0}^{\prime}<+\infty \text { and } l_{0} & =0 \Longrightarrow \lim _{0} \alpha=1 \\
l_{\infty}^{\prime}>0 \text { and } l_{\infty} & =+\infty \Longrightarrow \lim _{+\infty} \alpha=1
\end{aligned}
$$

\section{Proof. See appendix A.2. ${ }^{4}$}

This result can be stated in plain English the following way:

Let $f$ be a neoclassical production function. If factor shares do not tend to degenerated values in the neighborhood of infinity, then $f$ fulfills the Inada conditions near infinity. If factor shares do not tend to degenerated values in the neighborhood of zero, then $f$ fulfills the Inada conditions near zero.

Remarkably, the Inada conditions are compatible with ever-fluctuating factor shares. Indeed, asymptotic fluctuations in factor shares constitute a sufficient condition for $f$ to meet the Inada conditions - which also follows if factor shares converge to non-degenerated limits as the capitallabor ratio tends to infinity. In the next section we lay some examples of production functions pertaining to these different cases.

Let's call $\mathscr{I}$ the set of neoclassical production functions that satisfy the Inada conditions: ${ }^{5}$

$$
\mathscr{I}=\left\{f \in \mathscr{N} \mid \lim _{0} f=\lim _{\infty} f^{\prime}=0, \lim _{\infty} f=\lim _{0} f^{\prime}=\infty\right\}
$$

We also define a special subset of $\mathfrak{S}$ :

$$
\mathfrak{S}^{*}=\left\{\sigma \in \mathfrak{S} \mid\left(\int_{0}^{\bar{k}} \frac{\sigma-1}{\sigma} \frac{\mathrm{d} k}{k}\right) \text { and }\left(\int_{\bar{k}}^{\infty} \frac{\sigma-1}{\sigma} \frac{\mathrm{d} k}{k}\right) \text { do not diverge to }+\infty \text { nor to }-\infty\right\}
$$

The results of lemma 2 show that:

Theorem 2. Let $f \in \mathscr{N}$, and let $\sigma$ be the corresponding elasticity of substitution function. Then:

$$
\sigma \in \mathfrak{S}^{*} \Longrightarrow f \in \mathscr{I}
$$

The reciprocal of theorem 2 does not hold: there exists some production functions that fulfill the Inada conditions, with factor shares converging to degenerated values. ${ }^{6}$ For example, if $\sigma_{1}(k)=$ $\frac{\ln k}{\ln k-1}$ (for $k>\exp (1)$ ), then $\frac{\sigma_{1}-1}{\sigma_{1}}=\frac{1}{\ln k}$. A primitive of $1 / k \ln k$ is $\ln (\ln k)$, which tends to infinity as $k$ tends to infinity, and so $\lim _{k \rightarrow+\infty} \alpha(k)=1$. In virtue of theorem 1 , the corresponding functions $f_{1}$

\footnotetext{
${ }^{4}$ We are grateful to an anonymous referee for pointing out a mistake in the proof of this lemma in an earlier version of this paper

${ }^{5}$ Clearly, $\mathscr{I} \subset \mathscr{N}$ but $\mathscr{I} \neq \mathscr{N}$, since the CES, non-Cobb-Douglas production functions belong to $\mathscr{N}$ but not to $\mathscr{I}$.

${ }^{6}$ In the same vein, Palivos and Karagiannis (2010) proved that $\sigma \rightarrow 1$ is not a sufficient condition for Inada conditions to hold.
} 
all take the form: $f_{1}(k)=A \exp \left(\int_{\bar{k}}^{k} \frac{\mathrm{d} k^{\prime \prime}}{k^{\prime}\left(1+\frac{\exp \left(-\int_{k}^{k^{\prime}} \frac{\mathrm{d} k^{\prime \prime}}{k \ln k^{\prime \prime}}\right)}{\tilde{\Pi}}\right)}\right)$, with $\bar{k}>0, A>0$ and $\bar{\Pi}>0$. It is possible to analytically derive all functions $f_{1}$ :

$$
\begin{aligned}
f_{1}(k) & =A \exp \left(\int_{\bar{k}}^{k} \frac{\mathrm{d} k^{\prime}}{k^{\prime}\left(1+\frac{\exp \left(-\ln \left(\ln k^{\prime}\right)+\ln (\ln \tilde{k})\right)}{\bar{\Pi}}\right)}\right)=A \exp \left(\int_{\bar{k}}^{k} \frac{\mathrm{d} k^{\prime}}{k^{\prime}\left(1+\frac{\lambda}{\ln k^{\prime}}\right)}\right) \\
& =A \exp (\ln k-\lambda \ln (\lambda+\ln k)-c)
\end{aligned}
$$

with $\lambda=\ln \bar{k} / \bar{\Pi}>0$ and $c=\ln \bar{k}-\lambda \ln (\lambda+\ln \bar{k})$. Call $A^{\prime}=A \exp (-c)>0$, then $f_{1}$ admits the following analytical expression: $f_{1}(k)=A^{\prime} \frac{k}{(\lambda+\ln k)^{\lambda}}$. So $f_{1}(k) \stackrel{\infty}{\rightarrow} \infty$ as a consequence of power functions beating powers of logarithms near infinity. By differentiating the above expression, it comes: $f_{1}^{\prime}(k)=A^{\prime}\left(\frac{1}{(\lambda+\ln k)^{\lambda}}-\frac{\lambda}{(\lambda+\ln k)^{\lambda+1}}\right)=A^{\prime} \frac{\ln k}{(\lambda+\ln k)^{\lambda+1}}$, which proves that $f_{1}^{\prime} \stackrel{\infty}{\longrightarrow} 0$.

\subsection{The asymptotically-quasi-Cobb-Douglas production functions}

Robinson (1938) and Uzawa (1961) have shown that, in the canonical neoclassical growth framework, if the growth rate of real variables are constant then technical change has a labor-augmenting (LATC) representation, i.e. there exists a production function $F(\cdot, \cdot)$ such that output can be expressed like $Y_{t}=F\left(K_{t}, B_{t} L_{t}\right)$, where $B_{t}=B_{0} \exp \left(g_{B} t\right)$ is a steadily-increasing productivity term $\left(g_{B}>0\right)$. Then, output and capital both grow at the constant rate of $g_{B}$ along the stable equilibrium path. This result is known as the 'steady growth theorem'?

The Robinson-Uzawa theorem is probably the reason for the widespread representation of technical change as labor-augmenting. ${ }^{8}$ However, a whole strand of empirical literature, initiated by Greenwood et al. (1997), has firmly established that technical change was not correctly represented as labor-augmenting in the USA over the postwar period, and even less so since the early 1980's instead, technical change seems to be mostly embedded in more efficient (and/or cheaper) capital goods. In addition, it has been noted that the labor share has decreased steadily in most advanced countries between the late 1970's and the early 2010's, which also contradicts the LATC assumption. ${ }^{9}$

These empirical biases question the relevance of LATC-driven growth and raises the issue of whether the neoclassical growth framework can account for medium-run imbalances as well as for long-run regularities.

To do so, we investigate the conditions under which a simple neoclassical growth model yields asymptotically (rather than absolutely) constant growth rates. More precisely, we consider be-

\footnotetext{
${ }^{7}$ Jones and Scrimgeour (2008) interpret this result the following way: if there are several inputs to production, some accumulable and others not (e.g., capital vs. labor), then economic growth makes the accumulable inputs grow more quickly than the non-accumulable ones, except when technical change precisely increases the effective quantities of non-accumulable inputs. In the capital and labor inputs framework, technical change then has to be labor augmenting.

${ }^{8}$ Barro and Sala-i-Martín (2004, p. 53) reflect the common perception of the range of the Robinson-Uzawa theorem: "If we want to consider models that possess a steady state, we have to assume that technological progress takes the labor-augmenting form. (...) the long-term experiences of the United States and some other developed countries indicate that per capita growth rates can be positive and trendless over long periods of time (...). This empirical phenomenon suggests that a useful theory would predict that per capita growth rates approach constants in the long run; that is, the model would possess a steady state." We prove below that some neoclassical growth models can yield asymptotically constant ratios and growth rates without assuming that technological change is labor-augmenting nor that the production function is Cobb-Douglas.

${ }^{9}$ See de La Grandville (2009, chapter 5 co-written with Robert M. Solow) and Neiman and Karabarbounis (2014).
} 
low capital-augmenting (KATC) and investment-specific technical change (ISTC) - which both increase the effective quantity of capital - in a standard, continuous-time Solow model. We prove that the set of production functions for which growth is asymptotically balanced is exactly the set of production functions whose labor share function tends to some limit as the capital-labor ratio tends to infinity; a class of functions that we might dub the asymptotically-quasi-Cobb-Douglas production functions.

We denote by $\mathscr{Q}$ the set of production functions such that the capital share tends to some constant in $[0,1)$ as the effective capital-labor ratio tends to infinity:

$$
\mathscr{Q}=\left\{f \in \mathscr{N} \mid \lim _{x \rightarrow+\infty} \frac{x f^{\prime}(x)}{f(x)} \text { exists and } \in[0,1)\right\}
$$

For $f \in \mathscr{N}, f \in \mathscr{Q}$ if and only if the corresponding elasticity of substitution function $\sigma_{f}$ belongs to the set $\mathfrak{S}_{A}=\left\{\sigma \in \mathfrak{S} \mid \int_{\bar{k}}^{\infty} \frac{\sigma(k)-1}{\sigma(k)} \frac{\mathrm{d} k}{k} \in[-\infty,+\infty)\right\}$.

Definition 1. Let $\left(f_{t}\right)_{t \geq 0} \in \mathscr{N}^{[0,+\infty)}$ be a family of neoclassical production functions.A growth path is a specification of differentiable time paths $\left(k_{t}\right)_{t \geq 0}$ and $\left(y_{t}=f_{t}\left(k_{t}\right)\right)_{t \geq 0}$. A growth path is asymptotically balanced if and only if the growth rates of $k_{t}$ and $y_{t}$ (respectively $\dot{k}_{t} / k_{t}$ and $\dot{y}_{t} / y_{t}$ ) tend to positive constants $g_{k} \geq 0$ and $g_{y} \geq 0$ as $t$ tends to infinity.

Theorem 3. Let $s \in(0,1), \delta>0$ and $n \geq 0$ and let $f \in \mathscr{I}$.

- Let $\left(k_{t}, y_{t}\right)_{t \geq 0}$ be the growth path corresponding to the Solow model with steady KATC and LATC:

$$
\left\{\begin{array}{l}
y_{t}=B_{t} f\left(\frac{A_{t} k_{t}}{B_{t}}\right) \\
\dot{k}_{t}=s y_{t}-(\delta+n) k_{t}
\end{array}\right.
$$

with $\dot{A}_{t} / A_{t}=g_{A} \geq 0, \dot{B}_{t} / B_{t}=g_{B} \geq 0, k_{0}>0, A_{0}>0$ and $B_{0}>0$ given. Then:

$$
\left(k_{t}, y_{t}\right) \text { is asymptotically balanced } \Longleftrightarrow\left\{\begin{array}{l}
g_{A}=0 \\
o r \\
g_{A}>0 \text { and } f \in \mathscr{Q}
\end{array}\right.
$$

The asymptotic growth rates of $k$ and $y$ are then respectively: $g_{k}=g_{y}=g_{B}$ in the case $g_{A}=0$; and $g_{k}=g_{y}=g_{B}+\frac{\alpha_{\infty}}{1-\alpha_{\infty}} g_{A}$ in the case $g_{A}>0$ and $f \in \mathscr{Q}$, where $\alpha_{\infty}=\lim _{x \rightarrow+\infty} \frac{x f^{\prime}(x)}{f(x)}$.

- Let $\left(k_{t}, y_{t}\right)$ be the growth path corresponding to the Solow model with steady ISTC and LATC:

$$
\left\{\begin{array}{l}
y_{t}=B_{t} f\left(\frac{k_{t}}{B_{t}}\right) \\
\dot{k}_{t}=s q_{t} y_{t}-(\delta+n) k_{t}
\end{array}\right.
$$

with $\dot{B}_{t} / B_{t}=g_{B} \geq 0, \dot{q}_{t} / q_{t}=g_{q} \geq 0, k_{0}>0, A_{0}>0$ and $q_{0}>0$ given. Then:

$$
\left(k_{t}, y_{t}\right) \text { is asymptotically balanced } \Longleftrightarrow\left\{\begin{array}{l}
g_{q}=0 \\
o r \\
g_{q}>0 \text { and } f \in \mathscr{Q}
\end{array}\right.
$$

The asymptotic growth rates of $k$ and $y$ are then respectively: $g_{k}=g_{y}=g_{B}$ in the case $g_{q}=0$; and $g_{k}=g_{B}+\frac{1}{1-\alpha_{\infty}} g_{q}, g_{y}=g_{B}+\frac{\alpha_{\infty}}{1-\alpha_{\infty}} g_{q}$ in the case $g_{q}>0$ and $f \in \mathscr{Q}$, where $\alpha_{\infty}=\lim _{x \rightarrow+\infty} \frac{x f^{\prime}(x)}{f(x)}$. 
Proof. See appendix A.3.

Theorem 3 highlights the set of production functions, as well as the corresponding elasticity of substitution functions, such that the Solow growth model under KATC or ISTC yields asymptotically balanced growth. It comes out that these functions are exactly the functions in $\mathscr{Q}$. As such, theorem 3 constitutes an extension of the Robinson-Uzawa theorem, which implies that balanced (i.e. constant-rate) growth under KATC or ISTC is only possible if the production function is CobbDouglas. ${ }^{10}$

Notice that there are some production functions in $\mathscr{Q}$ such that the associated elasticity of substitution function does not tend to one near infinity. More precisely, it is possible that $f \in \mathscr{Q}$ with $\sigma$ oscillating around 1. Take, for instance, $\sigma_{2}(k)=\frac{1}{1-\frac{\sin k}{2}}$ for all $k \geq 0$, so that $\frac{\sigma_{2}(k)-1}{\sigma_{2}(k)}=\frac{\sin k}{2}$. Since function $x \mapsto \sin x / x$ is integrable near infinity, for any function $f_{2}$ corresponding to elasticity of substitution $\sigma_{2}$, the relative factor shares ratio $\Pi(k)$ tends to a strictly positive constant as $k$ tends to infinity. Still, $\sigma_{2}$ does not tend to one, as $\sigma_{2}$ oscillates periodically between $2 / 3$ and 2 .

The effective inputs ratio tends to a constant under pure LATC while it diverges under KATC/ISTC. Consequently, the production function influences growth dynamics in the KATC/ISTC-driven models, in which case theorem 3 proves that fluctuations of the labor share around a long-run mean is compatible with asymptotically balanced growth.

\section{Conclusion}

In this paper we have investigated the links between the set of 2-inputs neoclassical production function and the set of elasticity of substitution functions. In doing so we have unveiled an integral formula for all these production functions of class $\mathscr{C}^{2}$. We linked this formula to the Inada conditions and to the asymptotic balance of a standard growth model.

The generalization of this formula to the case of $n \geq 3$ inputs - which today is beyond our means - would be an answer to the question: 'how to mathematically represent constant-returnsto-scale aggregation processes?' and would as such represent a powerful tool for neoclassical analysis.

\section{A Appendix}

\section{A.1 2-input production functions in extensive and intensive forms}

Proposition. Let $F$ be a 2-input, constant returns to scale production function of class $\mathscr{C}^{2}$ in extensive terms: $F:\left(\mathbb{R}_{+}^{*}\right)^{2} \rightarrow \mathbb{R}_{+}^{*}$ and $f$ be the corresponding production function of class $\mathscr{C}^{2}$ in intensive

\footnotetext{
${ }^{10}$ Another extension found in the literature is due to Meade (1961, appendix II) who proved that a 2-sector growth model with Cobb-Douglas production functions for consumption and investment, when animated by steady consumption- and investment-specific TFP growth, is (strongly) balanced.
} 
terms: $f: \mathbb{R}_{+}^{*} \rightarrow \mathbb{R}_{+}^{*}$ defined by $\forall k \in \mathbb{R}_{+}^{*}, f(k)=F(k, 1)$. Then:

$$
\forall(K, L) \in\left(\mathbb{R}_{+}^{*}\right)^{2},\left\{\begin{array}{rl}
\frac{\partial F}{\partial K}(K, L) & >0 \\
\frac{\partial F}{\partial L}(K, L) & >0 \\
\frac{\partial^{2} F}{\partial K^{2}}(K, L) & <0 \\
\frac{\partial^{2} F}{\partial L^{2}}(K, L) & <0
\end{array} \Longleftrightarrow \forall k \in \mathbb{R}_{+}^{*}, f^{\prime}(k)>0, f^{\prime \prime}(k)<0 .\right.
$$

Proof. $\Longrightarrow$ Assume that the left-hand side conditions are fulfilled.

By the definition of $f, f(\cdot)=F(\cdot, 1)$ so $\forall k \in \mathbb{R}_{+}^{*}, f^{\prime}(k)=\frac{\partial F}{\partial K}(k, 1)>0$ and $\forall k \in \mathbb{R}_{+}^{*}, f^{\prime \prime}(k)=$ $\frac{\partial^{2} F}{\partial K^{2}}(k, 1)<0$.

$\Longleftarrow$ Assume that the right-hand side conditions are fulfilled.

Let $(K, L) \in\left(\mathbb{R}_{+}^{*}\right)^{2}$. By the definition of $f, F(K, L)=L f\left(\frac{K}{L}\right)$. Differentiating this identity with respect to $K$ twice yields:

$$
\begin{aligned}
\frac{\partial F}{\partial K}(K, L) & =f^{\prime}\left(\frac{K}{L}\right)>0 \\
\frac{\partial^{2} F}{\partial K^{2}}(K, L) & =\frac{1}{L} f^{\prime \prime}\left(\frac{K}{L}\right)<0
\end{aligned}
$$

Differentiating identity $F(K, L)=L f\left(\frac{K}{L}\right)$ with respect to $L$ yields: $\frac{\partial F}{\partial L}(K, L)=f\left(\frac{K}{L}\right)-\frac{K}{L} f^{\prime}\left(\frac{K}{L}\right)$. Since $f$ is assumed to be strictly positive and strictly increasing, $f$ admits a limit near $0^{+}$which we call $f(0) \in \mathbb{R}_{+}$. Then: $f(K / L)=f(0)+\int_{0}^{K / L} f^{\prime}(u) \mathrm{d} u$; so the latter identity can be re-written:

$$
\frac{\partial F}{\partial L}(K, L)=f(0)+\int_{0}^{\frac{K}{L}} f^{\prime}(u) \mathrm{d} u-\frac{K}{L} f^{\prime}\left(\frac{K}{L}\right)=f(0)+\int_{0}^{\frac{K}{L}}\left(f^{\prime}(u)-f^{\prime}\left(\frac{K}{L}\right)\right) \mathrm{d} u
$$

Since $f^{\prime}$ is strictly decreasing, then $\left(f^{\prime}(u)-f^{\prime}\left(\frac{K}{L}\right)\right)>0$ for all $u<K / L$, so $\int_{0}^{\frac{K}{L}}\left(f^{\prime}(u)-f^{\prime}\left(\frac{K}{L}\right)\right) \mathrm{d} u>$ 0 . Since $f(0) \geq 0$, we conclude that $\frac{\partial F}{\partial L}(K, L)>0$.

Differentiating the identity $F(K, L)=L f\left(\frac{K}{L}\right)$ twice with respect to $L$ yields:

$$
\frac{\partial^{2} F}{\partial L^{2}}(K, L)=-\frac{K}{L^{2}} f^{\prime}\left(\frac{K}{L}\right)+\frac{K}{L^{2}} f^{\prime}\left(\frac{K}{L}\right)-\frac{K}{L}\left(-\frac{K}{L^{2}}\right) f^{\prime \prime}\left(\frac{K}{L}\right)=\frac{K^{2}}{L^{3}} f^{\prime \prime}\left(\frac{K}{L}\right)<0 .
$$

It should be noted that the above computations show that for any neoclassical production functions in extensive terms of class $\mathscr{C}^{2} F$, the Hessian matrix of $F$ have a zero determinant and a strictly negative trace. The non-diagonal elements are:

$$
\frac{\partial^{2} F}{\partial K \partial L}=\frac{\partial}{\partial L}\left[f^{\prime}\left(\frac{K}{L}\right)\right]=-\frac{K}{L^{2}} f^{\prime \prime}\left(\frac{K}{L}\right)
$$

This leads to det $H=\frac{\partial^{2} F}{\partial K^{2}} \frac{\partial^{2} F}{\partial L^{2}}-\left(\frac{\partial^{2} F}{\partial K \partial L}\right)^{2}=0$ and $\operatorname{Tr} H=\frac{\partial^{2} F}{\partial^{2} K}+\frac{\partial^{2} F}{\partial^{2} L}<0$. The Hessian matrix is negative semi-definite and so function $F$ is globally concave. ${ }^{11}$

Constant-returns-to-scale functions in extensive terms are never strictly concave. Let $n \in \mathbb{N}^{*}$ and $F$ be a CRS function of $\mathbb{R}^{n}$ into $R$. Let $x \in \mathbb{R}^{n} \backslash\{(0, \cdots, 0)\}$ and let $\mu \in \mathbb{R} \backslash\{1\}$ and $y=\mu x$. Then, for any $\lambda \in(0,1)$ :

$$
F(\lambda x+(1-\lambda) y)=F((\lambda+\mu(1-\lambda)) x)=(\lambda+\mu(1-\lambda)) F(x)=\lambda F(x)+(1-\lambda) F(y)
$$

which proves that $F$ is not strictly concave.

\footnotetext{
${ }^{11}$ We acknowledge a referee for pointing out this result.
} 


\section{A.2 Proof of lemma 2}

- If $l_{0}>0$, then by the definition of $f$ in (4), function $k^{\prime} \mapsto \frac{1}{k^{\prime}\left(1+\frac{\exp \left(-\int_{k}^{k^{\prime}} \frac{\sigma-1}{\sigma} \frac{\mathrm{d} k^{\prime \prime}}{k^{\prime \prime}}\right)}{\tilde{\Pi}}\right)}$ is a (strictly) positive function that is integrable in the neighborhood of 0 . Since function $\left(\frac{1}{k^{\prime}}\right)$ is not integrable around $0^{+}$, in particular it must hold that: $\frac{1}{1+\frac{\exp \left(-\int_{k}^{k^{\prime}} \frac{\sigma-1}{\sigma} \frac{d k^{\prime \prime \prime}}{k^{\prime \prime}}\right)}{k^{\prime}}} \stackrel{\frac{k^{\prime} \rightarrow 0}{\longrightarrow}}{\longrightarrow}$. Hence: $\lim _{k \rightarrow 0} \Pi(k)=0$ and $\lim _{k \rightarrow 0} \alpha(k)=0$.

- If $l_{\infty}<+\infty$, then function: $k^{\prime} \mapsto \frac{1}{k^{\prime}\left(1+\frac{\exp \left(-\int_{\frac{k^{\prime}}{k}} \frac{\sigma-1}{\sigma} \frac{d k^{\prime \prime}}{k^{\prime \prime}}\right)}{\tilde{\Pi}}\right)}$ is positive and integrable around $+\infty$ and so, in particular, must be negligible compared to function $\left(\frac{1}{k^{\prime}}\right)$, which is not integrable near

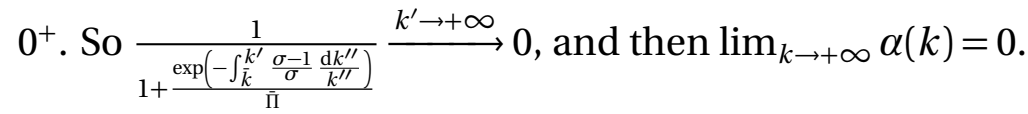

- If $l_{0}^{\prime}<+\infty$ and $l_{0}=0$, then by applying de L'Hôpital's rule we conclude that $\frac{f(k)}{k} \stackrel{k \rightarrow 0}{\longrightarrow} l_{0}^{\prime}$. But from equation (3), this implies that $\frac{1}{1+\frac{\exp \left(-\int_{\bar{k}}^{k^{\prime}} \frac{\sigma-1}{\sigma} \frac{\mathrm{d} k^{\prime \prime \prime}}{k^{\prime \prime}}\right)}{\tilde{\Pi}}} \stackrel{k^{\prime} \rightarrow 0}{\longrightarrow} 1$. Hence, $\lim _{k^{\prime} \rightarrow 0} \int_{\bar{k}}^{k^{\prime}} \frac{\sigma-1}{\sigma} \frac{\mathrm{d} k^{\prime \prime}}{k^{\prime \prime}}=+\infty$ and so $\lim _{k \rightarrow 0} \alpha(k)=1$.

- If $l_{\infty}^{\prime}>0$ and $l_{\infty}=+\infty$, then by de l'Hôpital's rule $\frac{f(k)}{k} \stackrel{k \infty}{\longrightarrow} l_{\infty}^{\prime}$ and so from equation (3): $\frac{1}{1+\frac{\exp \left(-\int_{\bar{k}}^{k^{\prime}} \frac{\sigma-1}{\sigma} \frac{1 k^{\prime \prime \prime}}{k^{\prime \prime}}\right)}{\bar{\Pi}}} \stackrel{k^{\prime} \rightarrow+\infty}{\longrightarrow}$ 1. So: $\lim _{k^{\prime} \rightarrow+\infty} \int_{\bar{k}}^{k^{\prime}} \frac{\sigma-1}{\sigma} \frac{\mathrm{d} k^{\prime \prime}}{k^{\prime \prime}}=+\infty$ and $\lim _{k \rightarrow+\infty} \alpha(k)=1$.

\section{A.3 Proof of theorem 3}

- Let's denote by $x_{t}$ the effective inputs ratio: $x_{t}=A_{t} k_{t} / B_{t}$. We rewrite equation (5) in terms of $\left(x_{t}, y_{t}\right)$ :

$$
\left\{\begin{array}{l}
y_{t}=B_{t} f\left(x_{t}\right) \\
\dot{x}_{t}=s A_{t} f\left(x_{t}\right)-\left(\delta+n-g_{A}+g_{B}\right) x_{t}
\end{array}\right.
$$

Differentiating the first equation yields:

$$
\frac{\dot{y}_{t}}{y_{t}}=g_{B}+\dot{x}_{t} \frac{f^{\prime}\left(x_{t}\right)}{f\left(x_{t}\right)}=g_{B}+\alpha_{t} \frac{\dot{x}_{t}}{x_{t}}
$$

where $\alpha_{t}=\alpha\left(x_{t}\right)=\frac{x_{t} f^{\prime}\left(x_{t}\right)}{f\left(x_{t}\right)}$ denotes the relative capital share at date $t$.

Then, by differentiating the second equation in (7) and re-injecting the same equation and the definition of the capital share, it comes that $\dot{x} / x$ follows the process:

$$
\stackrel{\stackrel{\dot{x}_{t}}{x_{t}}}{=}=\left(g_{A}-\left(1-\alpha\left(x_{t}\right)\right) \frac{\dot{x}_{t}}{x_{t}}\right)\left(\frac{\dot{x}_{t}}{x_{t}}+\delta+n-g_{A}+g_{B}\right)
$$

Note that the second term is positive, since $\dot{k}_{t} / k_{t} \geq-\delta-n$ at all dates. The differential equation of $\dot{x}_{t} / x_{t}(9)$ is not autonomous in $\dot{x} / x$ due to the term $\alpha\left(x_{t}\right)$.

We begin by proving a useful lemma:

Lemma 3. If $g_{A}>0$, then $x_{t} \stackrel{t \rightarrow+\infty}{\longrightarrow}+\infty$. 
Proof. We first show that $x_{t}$ is ultimately increasing, that is: $\exists \tau \geq 0 \mid \forall t \geq \tau, \dot{x}_{t} \geq 0$

- If $\delta+n-g_{A}+g_{B}<0$, then by the second equation in (7), $\dot{x}$ is positive at each date.

- If $\delta+n-g_{A}+g_{B}>0$, let's define for all $t$ the ratio $x_{t}^{*}$ such that

$$
s A_{t} f\left(x_{t}^{*}\right)=\left(\delta+n-g_{A}+g_{B}\right) x_{t}^{*}
$$

$x_{t}^{*}$ is the limit that $x_{t}$ would take if $A$ stayed constant at $A_{t}$ from date $t$ on. If $g_{A}>0$ then $x_{t}^{*}$ increases with time and $\lim _{t \infty} x_{t}^{*}=\infty$. By the second equation in (7):

$$
\dot{x}_{t} \lesseqgtr 0 \Longleftrightarrow x_{t} \gtreqless x_{t}^{*}
$$

* First case: suppose that $x_{0}<x_{0}^{*}$, so that $\dot{x}_{0}>0$. Suppose that there exists some $T>0$ such that $\dot{x}_{T}<0$, so $x_{T}^{*}<x_{T}$. Since $\dot{x}$ is a continuous function of time, we can define $\tau=\sup \left\{t_{1}<T \mid \dot{x}_{t_{1}} \geq 0\right\}$. Then, by continuity of $\dot{x}_{t}$,

$$
\begin{gathered}
\dot{x}_{\tau}=0 \\
\forall t \in(\tau, T], \dot{x}_{t}<0
\end{gathered}
$$

From (10), $x_{\tau}=x_{\tau}^{*}$. But since $\tau<T$, it comes that $x_{\tau}^{*}<x_{T}^{*}$. So $x_{\tau}=x_{\tau}^{*}<x_{T}^{*}<x_{T}$. But since $\tau<T$, (11) proves that $x_{\tau}>x_{T}$, which brings a contradiction.

So when $\dot{x}_{0}>0, \dot{x}_{t} \geq 0$ at all dates $t \geq 0$.

* Second case: suppose now that $x_{0} \geq x_{0}^{*}$, so that $\dot{x}_{0} \leq 0$. If $\dot{x}$ stays negative forever, then $x_{t}$ (which is then decreasing and positive) tends to a constant, while $\dot{x}_{t}$ then tends to zero; which is inconsistent with the second equation in (7) taken to the limit when $g_{A}>0$. So there exists $\tau>0$ such that $\dot{x}_{\tau}>0$. Then, by the same reasoning as in the first case above, $\dot{x}_{t} \geq 0$ for all $t \geq \tau$.

Then, since $x_{t}$ is ultimately increasing, $x_{t}$ either converges to a constant or diverges to $+\infty$. If $x_{t}$ converges to some constant $x_{l}$, then it converges monotonically to $x_{l}$ and so $\dot{x}_{t}$ must have limit 0 . But these two facts taken together are inconsistent with the second equation in (7) when $A_{t}$ increases steadily, so $x_{t} \stackrel{t \infty}{\longrightarrow}+\infty$.

We now proceed to the proof of the first part of theorem 3 concerning the Solow model under KATC and/or LATC.

$\Longrightarrow$ Suppose that $\frac{\dot{y}_{t}}{y_{t}}$ and $\frac{\dot{k}_{t}}{k_{t}}$ tend to constants $g_{y} \geq 0$ and $g_{k} \geq 0$, then by definition of $x$, $\frac{\dot{x}_{t}}{x_{t}}$ has limit $g_{k}+g_{A}-g_{B}$ as $t$ tends to infinity.

- If $g_{k}=g_{B}-g_{A}$ then $\frac{\dot{x}_{t}}{x_{t}} \stackrel{t \rightarrow+\infty}{\longrightarrow}$ 0. Equation (9) taken to the limit proves that $\stackrel{\stackrel{\dot{x}_{t}}{x_{t}}}{t \rightarrow+\infty}$ $g_{A}\left(\delta+n-g_{A}+g_{B}\right)=g_{A}\left(\delta+n+g_{k}\right)$. If $g_{A} \neq 0$ then the limit of $\frac{\dot{x_{x}}}{x_{t}}$ is stricly positive, which is inconsistent with the convergence of $\frac{\dot{x}_{t}}{x_{t}}$ to 0 . Thus, in this case, $g_{A}=0$.

- If $g_{k} \neq g_{B}-g_{A}$ then $\frac{\dot{x}_{t}}{x_{t}} \stackrel{t \rightarrow+\infty}{\longrightarrow} g_{k}+g_{A}-g_{B}$. Taking equation (8) to the limit proves that $\alpha_{t} \stackrel{t \rightarrow+\infty}{\longrightarrow} \frac{g_{y}-g_{B}}{g_{k}+g_{A}-g_{B}}$. 
Consequently, if $g_{A}>0$ then from lemma $2 x_{t} \stackrel{t \rightarrow+\infty}{\longrightarrow}+\infty$, so the function $x \mapsto \alpha(x)$ admits a limit when $x \rightarrow+\infty$. Call this limit $\alpha_{\infty}$. Equation (9) taken to the limit

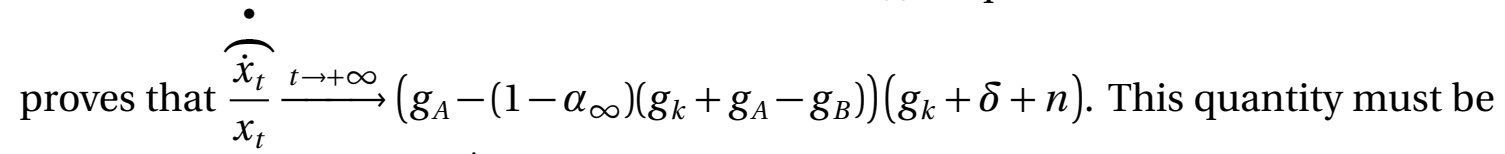
equal to zero, otherwise $\frac{\dot{x}_{t}}{x_{t}}$ does not converge. The only possibility is that we have $g_{A}=\left(1-\alpha_{\infty}\right)\left(g_{k}+g_{A}-g_{B}\right)$, which means that $\alpha_{\infty} \neq 1$ and $g_{k}=g_{B}+\frac{\alpha_{\infty}}{1-\alpha_{\infty}} g_{A}$. We get $g_{y}$ from equation (8): $g_{y}=g_{B}+\alpha_{\infty}\left(g_{k}+g_{A}-g_{B}\right)=g_{k}$. Finally, the limit value of $\alpha$ is $\frac{g_{y}-g_{B}}{g_{k}+g_{A}-g_{B}}=\alpha_{\infty} \in[0,1)$, which proves that $f \in \mathscr{Q}$.

$\Longleftarrow-$ If $g_{A}=0$, then $A_{t}=A_{0}$ for all $t \geq 0$, so the second equation in (7) is autonomous in $x_{t}$. Since $f$ satisfies the Inada conditions, $x_{t} \stackrel{t \rightarrow+\infty}{\longrightarrow} x^{*}$ characterized by $s A f\left(x^{*}\right)=$ $\left(\delta+n+g_{B}\right) x^{*}$ and $\dot{x}_{t} \stackrel{t \rightarrow+\infty}{\longrightarrow} 0$. From the definition of $x_{t}$ it comes that $k_{t} / B_{t}$ tends to a constant, and does monotonically, which proves that $\dot{k}_{t} / k_{t} \stackrel{t \rightarrow+\infty}{\longrightarrow} g_{B}$. Equation (8) proves that $\dot{y}_{t} / y_{t} \stackrel{t \rightarrow+\infty}{\longrightarrow} g_{B}$.

- If $g_{A}>0$ and $f \in \mathscr{Q}$, let's call $\alpha_{\infty}=\lim _{x \infty} x f^{\prime}(x) / f(x) \in[0,1)$. By lemma $3, \alpha_{t} \stackrel{t \rightarrow+\infty}{\longrightarrow}$ $\alpha_{\infty}$. The differential equation (9) then converges to the following one:

$$
\stackrel{\stackrel{\bullet}{\dot{x}_{t}}}{x_{t}}=\left(g_{A}-\left(1-\alpha_{\infty}\right) \frac{\dot{x}_{t}}{x_{t}}\right)\left(\frac{\dot{x}_{t}}{x_{t}}+\delta+n-g_{A}+g_{B}\right)=\Gamma\left(\frac{\dot{x}_{t}}{x_{t}}\right)
$$

which intercepts the horizontal axis at $g_{A} /\left(1-\alpha_{\infty}\right)$. Let $\varepsilon$ be a small, strictly positive number. Since $\alpha_{t}$ converges to $\alpha_{\infty} \in[0,1)$, then after some date $T_{1} \alpha_{t} \in\left(\alpha_{\infty}-\varepsilon, \alpha_{\infty}+\right.$ $\varepsilon)$.

Let's respectively denote by $\Gamma_{-}$and $\Gamma_{+}$the following functions:

$$
\begin{aligned}
& \Gamma_{-}(\chi)=\left(g_{A}-\left(1-\alpha_{\infty}+\varepsilon\right) \chi\right)\left(\chi+\delta+n-g_{A}+g_{B}\right) \\
& \Gamma_{+}(\chi)=\left(g_{A}-\left(1-\alpha_{\infty}-\varepsilon\right) \chi\right)\left(\chi+\delta+n-g_{A}+g_{B}\right)
\end{aligned}
$$

With these definitions, $\forall t \geq T_{1}: \Gamma_{-}\left(\frac{\dot{x}_{t}}{x_{t}}\right) \leq \Gamma\left(\frac{\dot{x}_{t}}{x_{t}}\right) \leq \Gamma_{+}\left(\frac{\dot{x}_{t}}{x_{t}}\right)$. So after $T_{1}, \dot{x}_{t} / x_{t}$ increases more than if it was guided by $\Gamma_{-}$and less than if it was guided by $\Gamma_{+}$. But the processes $\dot{\chi}=\Gamma_{-}(\chi)$ and $\dot{\chi}=\Gamma_{+}(\chi)$ converge respectively to $N=g_{A} /\left(1-\alpha_{\infty}+\varepsilon\right)$ and $O=g_{A} /(1-$ $\left.\alpha_{\infty}-\varepsilon\right)$. So there exists a date $T_{2} \geq T_{1}$ such that:

$$
\forall t \geq T_{2}, M=N-\varepsilon \leq \frac{\dot{x}_{t}}{x_{t}} \leq O+\varepsilon=P
$$

which proves that $\dot{x}_{t} / x_{t} \stackrel{t \rightarrow+\infty}{\longrightarrow} g_{A} /\left(1-\alpha_{\infty}\right)$. By the definition of $x, g_{k}=g_{B}+\frac{\alpha_{\infty}}{1-\alpha_{\infty}} g_{A}$ and by equation (8) $g_{y}=g_{B}+\frac{\alpha_{\infty}}{1-\alpha_{\infty}} g_{A}$.

- We denote by $u_{t}$ the effective inputs ratio: $u_{t}=k_{t} / B_{t}$. Differentiating the first equation in (6) yields:

$$
\frac{\dot{y}_{t}}{y_{t}}=g_{B}+\frac{\dot{u}_{t}}{u_{t}} \alpha\left(u_{t}\right)
$$

where $\alpha\left(u_{t}\right)=\frac{u_{t} f^{\prime}\left(u_{t}\right)}{f\left(u_{t}\right)}$.

The evolution of $u_{t}$ is guided by:

$$
\dot{u}_{t}=s q_{t} f\left(u_{t}\right)-\left(\delta+n+g_{B}\right) u_{t}
$$


Differentiating equation (14) and re-injecting the same equation and the definition of the capital share yields:

$$
\frac{\dot{\dot{u}_{t}}}{u_{t}}=\left(\frac{\dot{u}_{t}}{u_{t}}+\delta+n+g_{B}\right)\left(g_{q}-\left(1-\alpha\left(u_{t}\right)\right) \frac{\dot{u}_{t}}{u_{t}}\right)
$$

The same way as in the KATC case, we can prove the following lemma:

Lemma 4. If $g_{q}>0$ then $u_{t} \stackrel{t \rightarrow+\infty}{\longrightarrow}+\infty$.

Proof. Same method as in the proof of lemma (2) applied to (14).

We know prove the assertions of the second part of theorem 3 concerning the Solow model under ISTC and/or LATC with the same method as in the KATC/LATC case.

$\Longrightarrow$ Suppose that $\dot{y} / y \rightarrow g_{y} \geq 0$ and $\dot{k} / k \rightarrow g_{k} \geq 0$, then by equation (13) $\dot{u} / u \rightarrow g_{k}-g_{B}$.

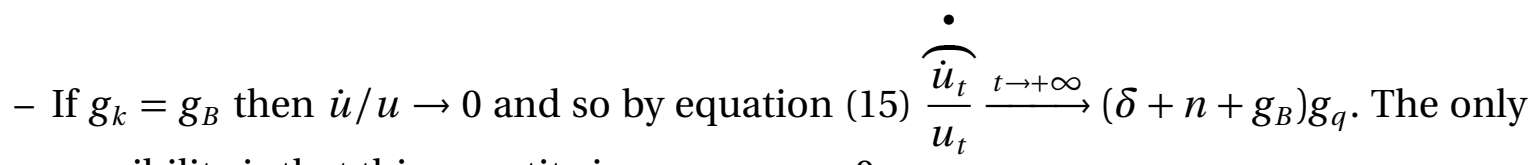
possibility is that this quantity is zero, so $g_{q}=0$.

- If $g_{k} \neq g_{B}$ then by (15) $\alpha_{t} \stackrel{t \rightarrow+\infty}{\longrightarrow} \frac{g_{y}-g_{B}}{g_{k}-g_{B}}$. Consequently, if $g_{q}>0$ then from lemma 4 $u_{t} \stackrel{t \rightarrow+\infty}{\longrightarrow}+\infty$, so function $u \mapsto \alpha(u)$ admits a limit as $u$ tends to infinity. Call this limit $\alpha_{\infty}$. Equation (15) taken to the limit proves that $\stackrel{\stackrel{\bullet}{\dot{u}_{t}}}{u_{t}} \stackrel{t \rightarrow+\infty}{\longrightarrow}\left(g_{k}+\delta+n\right)\left(g_{q}-\left(1-\alpha_{\infty}\right)\left(g_{k}-g_{B}\right)\right)$.

But since $\frac{\dot{u}_{t}}{u_{t}}$ converges, the limit of $\frac{\dot{\dot{u}_{t}}}{u_{t}}$ must be zero, which proves that $g_{q}-(1-$ $\left.\alpha_{\infty}\right)\left(g_{k}-g_{B}\right)=0$, so $\alpha_{\infty}<1$, which achieves to prove that $f \in \mathscr{Q}$. Also $g_{k}=g_{B}+\frac{1}{1-\alpha_{\infty}} g_{q}$ and equation (13) taken to the limit proves that $g_{y}=g_{B}+\frac{\alpha_{\infty}}{1-\alpha_{\infty}} g_{q}$.

$\Longleftarrow-$ If $g_{q}=0, u_{t}$ follows the autonomous, stable process $\dot{u}_{t}=s q_{0} f\left(u_{t}\right)-\left(\delta+n+g_{B}\right) u_{t}$, so $u_{t}$ converges to some limit $u^{*}>0$ and the growth path is asymptotically balanced, with $g_{k}=g_{y}=g_{B}$.

- If $g_{q}>0$ and $f \in \mathscr{Q}$, the the process for $\dot{u}_{t} / u_{t}$ converges to the following one:

$$
\frac{\dot{\dot{u}_{t}}}{u_{t}}=\left(\frac{\dot{u}_{t}}{u_{t}}+\delta+n+g_{B}\right)\left(g_{q}-\left(1-\alpha_{\infty}\right) \frac{\dot{u}_{t}}{u_{t}}\right)
$$

With the same method than in the Solow model under KATC above, this is straightforward to prove that $\dot{u}_{t} / u_{t} \rightarrow \frac{g_{q}}{1-\alpha_{\infty}}$. So $\dot{k}_{t} / k_{t} \stackrel{t \infty}{\longrightarrow} g_{B}+\frac{g_{q}}{1-\alpha_{\infty}}$ and from equation (13), $\dot{y}_{t} / y_{t} \stackrel{t \infty}{\longrightarrow} g_{B}+g_{q} \frac{\alpha_{\infty}}{1-\alpha_{\infty}}$.

\section{References}

[1] K. J. Arrow, H. B. Chenery, B. S. Minhas and R. M. Solow (1961) "Capital-Labor Substitution and Economic Efficiency,” Review of Economics and Statistics, Vol. 43, No. 3 (Aug.), pp. 225250. 
[2] R. BArro and X. SAlA-I-MARTÍN (2004) Economic Growth, Cambridge: MIT Press (first edition in 1995, New York: McGraw-Hill).

[3] O. De La GrandVILle (2009) Economic Growth - A Unified Approach, Cambridge University Press.

[4] J. Greenwood, Z. Hercowitz and P. Krusell (1997) "Long-Run Implications of InvestmentSpecific Technological Change," American Economic Review, Vol. 87, No. 3 (Jun.), pp. 342-362.

[5] J. Greenwood and M. Yorukoglu (1997) “1974,” Carnegie-Rochester Conference Series on Public Policy, Vol. 46, No. 1 (Jun.), pp. 49-95.

[6] C. I. Jones and D. Scrimgeour (2008) "A New Proof of Uzawa's Steady-State Growth Theorem," Review of Economics and Statistics, Vol. 90, No. 1 (Feb.), pp. 180-182.

[7] L. Karabarnounis and B. NeIman (2014) “The Global Decline of the Labor Share,” Quarterly Journal of Economics, Vol. 129, No. 1 (Feb.), pp. 61-103.

[8] J. MEADE (1961) A Neo-Classical Theory of Economic Growth, London: Allen \& Unwin.

[9] T. Palivos and G. Karagiannis (2010) "The Elasticity of Substitution as an Engine of Growth," Macroeconomic Dynamics, Vol. 14, No. 5 (Nov.), pp 617-628.

[10] N. S. Revankar (1971) "A Class of Variable Elasticity of Substitution Production Functions," Econometrica, Vol. 39, No. 1 (Jan.), pp. 61-71.

[11] J. Robinson (1938) “The Classification of Inventions," Review of Economic Studies, Vol. 5, No. 2 (Feb.), pp. 139-142.

[12] H. UZAWA (1961) "Neutral Inventions and the Stability of Growth Equilibrium," Review of Economic Studies, Vol. 28, No. 2 (Feb.), pp. 117-124. 